\title{
Exploiting Topology and Behavioral Attributes for Effective Routing in Mobile Networks
}

\author{
Ioannis Manolopoulos*†, Kimon Kontovasilis*, Ioannis Stavrakakis ${ }^{\dagger}$ and Stelios C. A. Thomopoulos* \\ *Institute of Informatics \& Telecommunications, N.C.S.R. Demokritos, \\ P.O. Box 60228, GR-15310 Ag. Paraskevi, Greece. Email: \{imanolo,kkont,scat\}@iit.demokritos.gr \\ ${ }^{\dagger}$ Department of Informatics \& Telecommunications, National \& Kapodistrian University of Athens, \\ GR-15784 Ilissia, Greece. Email: ioannis@di.uoa.gr
}

\begin{abstract}
In this paper we explore the interplay of node density, mobility and cooperation in routing decisions for mobile networks. Specifically, routing is based on a novel metric that jointly accounts for all three aforementioned attributes and helps identify the most promising venue available for getting the message closer/faster to the destination, even in dynamically changing environments. The effectiveness of the proposed scheme is validated through simulations.
\end{abstract}

\section{INTRODUCTION}

The future-internet vision includes scenarios where various topologies involving different devices are frequently associated with uneven network density and mobility conditions that may vary widely. Moreover, social relations might introduce additional nodal dependencies that can increase the variability of conditions even further. However, most current routing protocols function satisfactorily only under certain presupposed fixed conditions.

In classifying the diversity of topological conditions in networking environments, one end of the spectrum corresponds to well-connected dense topologies, where protocols based on the forward action (i.e., the spatial transposition of messages due to hops from one node to another) function best. This category includes both of the topology-based and position-based families of protocols. Protocols in the first family are suitable for topologies with relatively static nodes and use only node identities. Protocols in the second family [1] can cope with more mobile nodes (provided this mobility does not affect the network connectivity) and, in addition to identities, employ position information too.

The other side of the spectrum corresponds to networks with relatively low density, where intense mobility is a prerequisite for the creation of contact opportunities. For such topologies, protocols based on the carry action [2] (i.e., the spatial transposition of the message due to the physical movement of the carrier node) are better. They employ information about the nodes' mobility characteristics.

The various routing categories just reviewed are depicted in Fig. 1.a). Clearly, routing protocols capable of coping with diverse conditions should resort to an appropriate, dynamically adjusted combination of both

This work has been supported in part by the project GREENET EU FP7-PEOPLE-2010-ITN-264759 and the Network of Excellence in Internet Science project EINS (FP7-IST- 288021). the forward and carry actions. This has been addressed by the Maximum Advance Decision-MAD protocol [3], which employs position and velocity information for the calculation of the, so called, advance metric. This metric uses an estimation of the retaining time, namely the time a next-hop candidate node will retain the message, if selected. The retaining time reflects the relative importance of the forwarding and carry actions, towards adapting the protocol's operation under varying mobility and node density conditions. The original MAD [3] suggests a simple way to calculate the retaining time, which, despite being successful under many conditions, falls short of taking fully into account the entirety of the available density and mobility information. The first contribution of this paper is an improved estimation of the retaining time.

Additionally, we consider social ties (trustworthiness, friendship, willingness) of varying strength between nodes [4]. Proposals for forwarding strategies in such environments improve over pure contact-based metrics by incorporating social tie parameters from higher layers through simple heuristics [5]. Towards the second contribution in this paper, it is shown that aspects of node cooperation can be captured more precisely through the notion of density. This is achieved by means of subjective density, whose value depends not only on the topological density but also on each node's cooperation characteristic.

\section{The MAD Routing Protocol}

We consider a mobile ad hoc setting, where $R$ stands for the transmission range of each mobile node. A node possessing a packet, called the current node, periodically checks its neighborhood, evaluates the appropriateness of nodes therein as a next hop for the packet and forwards the packet to the most suitable such neighbor. If the current node approaches the destination node within range, it immediately delivers the packet to it.

The neighbor nodes are evaluated by means of the $a d-$ vance metric, which assesses (in units of speed) the rate at which the packet will approach its destination, if the node associated with the metric is selected as the packet's next hop. High rates of approach contribute to a smaller end-toend delay. The metric can capture the combined effect of the location and motion attributes of the associated node. In the following we denote the distance at time $t$ between two nodes $i$ and $j$ as $d_{i j}(t)$. The reduction of a packet's 
distance (actually, the carrier node's distance) from its destination is called progress and is measured in units of length. The overall progress is the sum of two components. The first is the progress due to the forward action (which is assumed to occur instantaneously at time $t=0$ ) from current node $C$ to the neighbor $i$, i.e., $d_{C D}(0)-d_{i D}(0)$. The second occurs from the subsequent carry action. The effect of this action is $d_{i D}(0)-d_{i D}\left(T_{i}\right)$ and depends on the time $T_{i}$ that node $i$ will retain the packet if it is selected as the next hop node. Note that when node $i$ keeps on moving on a straight line trajectory at constant speed, its distance from the destination as a function of time is simply $d_{i D}(t)=\sqrt{\left(V_{i} t\right)^{2}+d_{i D}(0)^{2}-2 V_{i} t d_{i D}(0) \cos \phi_{i}}, \quad \forall t \geq 0$.

The overall progress occurs over time $T_{i}$. Therefore, given an estimation of this retaining time yields an advance metric equal to

$$
\mathrm{ADV}_{C i, \text { tot }}=\frac{d_{C D}(0)-d_{i D}\left(T_{i}\right)}{T_{i}} .
$$

The selection of node $i$ is worthwhile only if $\mathrm{ADV}_{C i \text {,tot }}$

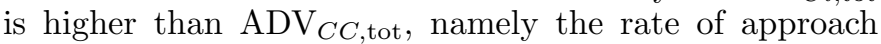
that will be achieved when the current node retains the message. This second rate is due only to the carry effect and is calculated setting $i=C$ in (1), but maintaining the same value of the retaining time $T_{i}$ used when calculating $\mathrm{ADV}_{C i \text {,tot }}$ (the reason being that the advance of both nodes should be determined over the same time interval). The relative merit of the neighbor node $i$ over the current node $C$ is expressed as the difference

$$
\Delta_{i}=\mathrm{ADV}_{C i, \text { tot }}-\mathrm{ADV}_{C C, \text { tot }}=\frac{d_{C D}\left(T_{i}\right)-d_{i D}\left(T_{i}\right)}{T_{i}} .
$$

If $\Delta_{i}>0$, node $i$ is more beneficial than the current node and becomes a next hop candidate. The same procedure is repeated for all neighbors of the current node and ultimately the packet is forwarded to the node with index $j=\arg \max _{i} \Delta_{i}$, justifying the name of the protocol (as Maximum Advance Decision).

\section{Estimating the Retaining Time}

The retaining time is a key-factor contributing to the effectiveness of the advance metric, so its proper estimation is an integral part of the protocol. However, the estimation method presented in the original MAD protocol [3] fails to fully reflect the fact that, regardless of the direction of motion, as the forwarding opportunities (neighbors) observed by the node per unit of time increase, due to either an increased density $\rho$ (equal to the number of neighbors divided by the area of the coverage region $\pi R^{2}$ ) and/or an increased speed $V_{i}$, the retaining time of this node will tend to become smaller. Therefore, one direction for improvement over the already presented estimation is to involve both of the density and speed parameters in the retaining times relevant to all directions of motion. Additionally, a node moving towards the destination can be treated in a more refined way, by distinguishing between directions enabling the node to approach the destination close enough for delivering the message itself and directions for which such delivery is not possible. We use $V_{i}$ and $\phi_{i}$ to denote the magnitude of node's $i$ velocity and the angle between this velocity and the line segment from node $i$ to the destination $D$, respectively, at time $t=0$.

For the case of moving away from the destination $\left(\cos \phi_{i} \leq 0\right)$ the motion is counterproductive and the node should find a next hop to forward the packet as soon as possible. The refinement is achieved by setting the smallest possible retaining time (corresponding to $\rho=\rho_{\max }$ ) equal to the time required by the node for renewing its neighborhood, namely equal to $R / V_{i}$ (first branch in (3)).

When the node moves towards the destination $\left(\cos \phi_{i}>0\right)$, it will eventually become capable of delivering the message to it exactly when $\cos \phi_{i} \geq \sqrt{1-\left[R / d_{i D}(0)\right]^{2}}$. The destination will enter the node's range and receive the message at time $T_{i, \max }=\left(d_{i D}(0) \cos \phi_{i}-\right.$ $\left.\sqrt{R^{2}-\left[d_{i D}(0) \sin \phi_{i}\right]^{2}}\right) / V_{i}$ (see Fig. 1.b)). This is the greatest possible value for the retaining time in this case and it is assumed to occur in environments with the smallest expected density $\rho_{\min }$ (middle branch of $(3)$ ).

Finally, for motion towards the destination without the possibility of direct delivery $\left(0<\cos \phi_{i}<\sqrt{1-\left[R / d_{i D}(0)\right]^{2}}\right), \quad$ the node will keep approaching the destination up to time $T_{i \text {, ben }}=d_{i D}(0) \cos \phi_{i} / V_{i}$, namely the time required for node $i$ to reach the point closest to the destination along its straight-line trajectory. Subsequently, it will move away (third branch in $(3)$ ).

For all the three cases mentioned above it holds that the more neighbors around node $i$, the easier it becomes to find a next hop, thus the time required for that is an inversely proportion of the node's local density $\rho$. From the previous discussion one obtains:

$$
\hat{T}_{i}= \begin{cases}\frac{R}{V_{i}} \frac{\rho_{\max }}{\rho}, & \cos \phi_{i} \leq 0, \\ T_{i, \max \frac{\rho_{\min }}{\rho},} & \cos \phi_{i} \geq \sqrt{1-\left[\frac{R}{d_{i D}(0)}\right]^{2}}, \\ T_{i, \text { ben }} \frac{\rho_{\min }}{\rho}+\frac{R}{V_{i}} \frac{\rho_{\max }}{\rho}, & \text { otherwise. }\end{cases}
$$

When evaluating (2), and in order to determine a common time for both terms of the difference $\Delta_{i}$, the estimate $\hat{T}_{C}$ is also calculated, by applying (3) with $i=C$, and the value $T_{i}=\min \left\{\hat{T}_{C}, \hat{T}_{i}\right\}$ is employed.

\section{Cooperation Awareness Routing}

The number of forwarding opportunities observed by a node at each point in time is a key parameter influencing the node's retaining time and, through it, the efficiency of the routing protocol. Up until now the forwarding opportunities have been quantified through the topological density. However, individual characteristics of the nodes inherited from higher layers may influence a node's perception about the observed forwarding opportunities.

Such characteristics of a node (e.g. trustworthiness, friendship, willingness) can be represented through an abstract metric that indicates the cooperation level of that node in a specific local routing environment. The cooperation level, $c$, takes values in the interval [0,1], where 1 indicates complete cooperation and 0 indicates complete lack of cooperation. By means of $c_{i}$, node $i$ distinguishes the neighbors that exist in a routing environment (namely 


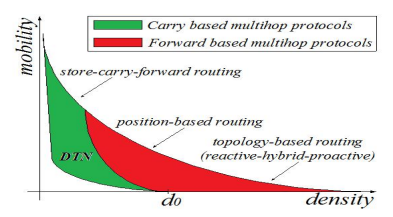

a.

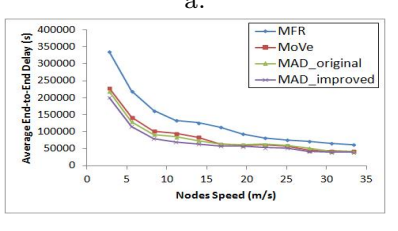

e. e2e delay in low density

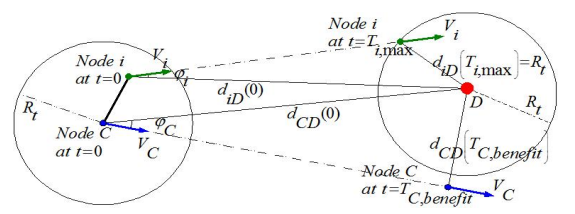

b.

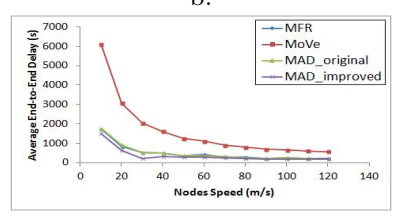

f. e2e delay in high density

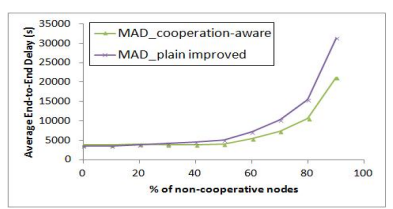

c. e2e delay

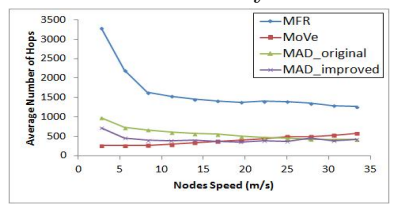

g. \# hops in low density

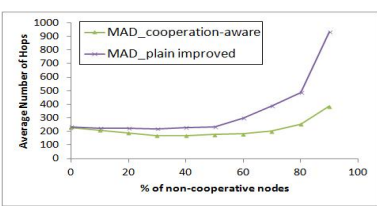

d. \# hops

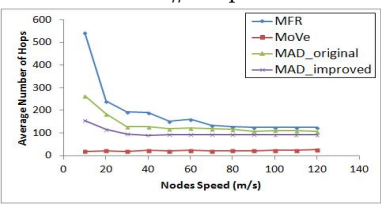

h. \# hops in high density

Fig. 1. a) Network Conditions and Routing Classes b) Forward and Carry Actions c)-d) Average e2e delay and \# hops vs non-cooperative environment (500 nodes and $120 \mathrm{~km} / \mathrm{h}$ ) e)-h) Average e2e delay and \# hops vs speed for low (75 nodes) and high (4750 nodes) density.

the topological density $\rho$, which is the same for all nodes in the same region) from the number of forwarding opportunities this individual node observes in the said environment. This second quantity is called subjective density and its value is $s d_{i}=c_{i} \rho$. The individual characteristics of nodes can then be incorporated in the decision policy of the MAD, in a non-arbitrary and robust way, simply by using $s d_{i}$ in place of $\rho$ in $(3)$.

\section{Evaluation Results}

We present results on the OPNET Modeler network simulator. The simulations addressed a network topology where the mobile nodes, with $R=250 \mathrm{~m}$, moved according to the random way-point mobility model with zero pause time and constant speed within a rectangular open area of size $10 \mathrm{~km} \times 10 \mathrm{~km}$. The source and destination were static nodes at diagonally opposite corners of the rectangle. The performance metrics were the end-to-end delay and the number of hops required for message delivery.

To assess the improvement in the retaining time estimation we compare the performance of the MAD routing protocol using the improved retaining time estimation (labeled "improved MAD") with three other protocols namely the forward-based greedy MFR protocol [1], the carry-based MoVe protocol [2] and the MAD protocol with the original expressions for the retaining time [3] (labeled "original MAD"). A wide range of speed values were examined, from $2.8 \mathrm{~m} / \mathrm{s}$ to $33.3 \mathrm{~m} / \mathrm{s}$ in incremental steps of $2.8 \mathrm{~m} / \mathrm{s}$. For each value of speed, a very sparse topology with 75 nodes in the rectangular area and a very dense topology with 4750 nodes in the area were examined (these values used in (3) for $\rho_{\min }$ and $\rho_{\max }$ respectively).

Fig. 1.e)-h) display end-to-end delays and hop counts, respectively, as a function of nodes' speed, for low and high densities. It can be evidenced that MAD exhibits the best performance, regardless of conditions, compared with the MFR and MoVe because it simultaneously takes advantage of both the forward and carry actions. Moreover, the improved MAD outperforms the original MAD protocol because of the more accurate retaining time estimation.

For the subjective density, two versions of improved MAD were considered: One ("plain improved MAD") with the topological density $\rho$, as previously, and another ("cooperation-aware MAD") with the subjective density sd instead. A topology of moderate density (500 nodes in the rectangular area) and intense mobility (speed 120 $\mathrm{km} / \mathrm{h}$ ) was tested. Various environments of widely varying degrees of cooperation were examined, from completely cooperative to $90 \%$ of the nodes non-cooperative, varying the percentage of the total nodes being non-cooperative in incremental steps of $10 \%$. A node was characterized cooperative if the value of its cooperation level $(c)$ ranged between 0.8 and 1 and non-cooperative if the value of its cooperation level $(c)$ ranged between 0 and 0.2 . The actual value was sampled uniformly in each range.

Fig. 1.c)-d) display end-to-end delays and hop counts, respectively, as a function of the percentage of the total nodes that are non-cooperative. The results provide direct evidence that the cooperative-aware MAD protocol becomes more efficient compared to the 'plain' improved MAD protocol, because the cooperation aspects are now taken into account through the subjective density.

\section{Conclusions}

In this paper we have explored the interplay of node density, mobility and cooperation in routing decisions in mobile networks. The first contribution relates to a redesign of the Maximum Advance Decision (MAD) mechanism. The paper has also introduced the novel notion of subjective density which couples the topological density with higher layer properties relevant to individual nodes, such as cooperation attributes.

\section{REFERENCES}

[1] H. Takagi and L. Kleinrock, "Optimal transmission ranges for randomly distributed packet radio terminals," IEEE Trans. Commun., vol. 32, pp. 246-257, mar 1984.

[2] J. Lebrun, C.-N. Chuah, D. Ghosal, and M. Zhang, "Knowledgebased opportunistic forwarding in vehicular wireless ad hoc networks," in VTC'05, may-june 2005.

[3] I. Manolopoulos, K. Kontovasilis, I. Stavrakakis, and S. Thomopoulos, "Mad: A dynamically adjustable hybrid location-and motion-based routing protocol for vanets," in $I S W C S$ '10, sept. 2010, pp. 696-700.

[4] E. Daly and M. Haahr, "Social network analysis for information flow in disconnected delay-tolerant manets," IEEE Trans. Mobile Comput., vol. 8, pp. 606-621, may 2009.

[5] Q. Li, W. Gao, S. Zhu, and G. Cao, "A routing protocol for socially selfish delay tolerant networks," Ad Hoc Networks, vol. 10, pp. 1619-1632, 2012. 